\title{
ANÁLISIS DE LAS POLÍTICAS PÚBLICAS AGRÍCOLAS Y LA SEGURIDAD ALIMENTARIA DE LA PROVINCIA DE LOS RÍOS
}

\section{ANALYSIS OF AGRICULTURAL PUBLIC POLICIES AND FOOD SECURITY IN THE PROVINCE OF LOS RÍOS}

\begin{abstract}
Katty Pilar Jadán Solís, Mgtr. Magíster en Economía mención en Desarrollo Económico y Políticas Públicas (Ecuador). Docente Universidad Técnica de Cotopaxi, La Maná, Ecuador. katty.jadan@utc.edu.ec
\end{abstract}

Rodolfo Najarro Quintero, Mgtr. Magíster en Conectividad y Redes de Ordenadores (Ecuador). Docente de la Universidad Técnica de Cotopaxi, La Maná, Ecuador. rodolfo.najarro@utc.edu.ec

\section{ARTÍCULO DE INVESTIGACIÓN}

Recibido: 7 de septiembre de 2019.

Aceptado: 12 de octubre de 2019.

\section{RESUMEN}

En el trabajo se aborda el estudio sobre los diferentes problemas relacionados con la seguridad alimentaria en Ecuador, uno de ellos es que se produce alimentos suficientes para satisfacer la demanda interna, tomando en cuenta que a lo largo de esta década el crecimiento de la producción agrícola es de 4,9\% lo cual es muy superior al de la población, que su crecimiento se estima de 1,5\%. A pesar que el $8,7 \%$ de hogares ecuatorianos no cuenta con los medios para tener acceso a una cantidad de alimentos suficientes para cubrir sus necesidades mínimas. El objetivo de la presente investigación es analizar las políticas públicas agrícolas encaminadas a garantizar la seguridad alimentaria de la Provincia. En el proceso de investigación se realizó el estudio de las variables de la dimensión económica que caracterizan el contexto de desarrollo territorial, destacando las referidas a la actividad agrícola y con ello las potencialidades locales, recursos y potestades administrativas para la gestión estratégica a este nivel, 
por lo que se efectuó un estudio correlacional determinando el nivel de especialización de la producción agrícola, analizando los rendimientos productivos existentes en la zona, identificando los factores determinantes de la producción agrícola como elementos de política pública y la proyección estratégica del desarrollo económico del sector.

Palabras clave: Políticas Públicas, seguridad Alimentaria, Administración Pública.

\section{ABSTRACT}

The paper addresses the study on the different problems related to food security in Ecuador, one of which is that enough food is produced to meet domestic demand, taking into account that throughout this decade the growth of agricultural production it is $4.9 \%$ which is much higher than the population, whose growth is estimated at $1.5 \%$. Although $8.7 \%$ of Ecuadorian households do not have the means to access a sufficient amount of food to cover their minimum needs. The objective of the present investigation is to propose a design of territorial public policies aimed at guaranteeing the food security of the Province. In the research process, the variables of the economic dimension that characterize the territorial development context were studied, highlighting those related to agricultural activity and with it the local potential, resources and administrative powers for strategic management at this level, Therefore, a correlation study was carried out determining the level of territorial specialization of agricultural production, analyzing the productive yields existing in the area, identifying the determinants of agricultural production as elements of public policy and the strategic projection of the economic development of the sector.

Keywords: Public politics, food safety, Public administration.

\section{INTRODUCCIÓN}

La problemática de la seguridad alimentaria ha adquirido relevancia en la sociedad actual y continúa su auge; en esta temática son protagonistas, en mayor medida, los países con dificultades socioeconómicas o áreas de población con difícil acceso a

ISSN: 1390-9320, Edición Especial, diciembre 2019 
recursos; ya sean productivos, asociados a procesos de elaboración de alimentos o condicionados por factores económicos - financieros o recursos dinerarios encargados de la financiación alimentaria, fenómeno que aparece tanto en el ámbito público, como privado.

Alineado a lo anterior puede atribuirse, cada vez más, el visible incremento de las poblaciones versus el decrecimiento de las fuerzas productivas agropecuarias, las situaciones climatológicas o fenómenos naturales que amenazan la producción de alimentos, la concentración de la propiedad, la dominación del mercado, entre otros factores; observando que el papel de la administración pública es vital en la mejora de los procedimientos a seguir, los recursos a destinar y las indicaciones de prioridad en función de los objetivos.

Afrontar los desafíos de la problemática alimentaria, es la meta principal del segundo objetivo de Desarrollo Sostenible de la Agenda 2030: asegurar el acceso de todas las personas a una alimentación sana, nutritiva y suficiente como se encuentra detallado en la meta 2.1 y eliminar todas las formas de malnutrición según la meta 2.2; prescrito por el CEPAL, aunque para lograr ello se deban articular otros objetivos propuestos.

En este contexto se presenta la problemática en el Ecuador, asociado a la necesidad de desarrollar una autosuficiencia alimentaria en correspondencia con las propias potencialidades de los territorios, donde quizás no se cuente con condiciones regionales para convertir a provincias y cantones como autosuficientes en materia alimentaria, pero pueden generarse procesos de asociatividad y mancomunidad entre estas, en función de articular estrategias, procesos productivos y de servicios, infraestructura, entre otros, que conduzcan a mejorar paulatinamente las políticas de seguridad alimentaria en la nación.

Para lograr el desarrollo sostenible es fundamental garantizar una vida saludable y promover el bienestar para todos a cualquier edad. Se han obtenido grandes progresos en relación con el aumento de la esperanza de vida y la reducción de algunas de las causas de muerte más comunes relacionadas con la mortalidad infantil y materna. (CEPAL, Metas del Objetivo 2, 2016, p. 13) 
En otro orden, asociado al ámbito público, no se cuenta con mecanismos que hayan certificado un modelo de gestión agrícola, a pesar que, como representantes de los intereses del conjunto de la sociedad, estos entes deberían integrar en su gestión este tipo de estándares. Por esto, y para brindar una posible respuesta a las preocupaciones crecientes de la sociedad en materia alimenticia, es recurrente una estrategia y a su vez, una política, consecuente con una serie de oportunidades que se observaron durante el proceso de revisión inicial, enfocadas hacia la optimización de los procesos productivos agrícolas, para que en los marcos de la gobernabilidad provincial se implemente y aplique.

La necesidad de que el estudio se realice en la provincia Los Ríos, parte de la problemática que enmarca el sector agrícola desde año 2015 en este territorio. Según análisis realizado por el MAGAP (Ministerio de agricultura y Ganadería) del Ecuador. En primer lugar, aparece la falta de legalización de tenencia de tierra. La falta de tecnificación y maquinaria para los pequeños productores, así como también la implementación de más centros de acopio son los principales problemas estructurales que adolecen a los agricultores. Sin dejar de mencionar una política de crédito que sea eficiente y oportuna.

Otro problema estructural es la escasa cultura de compromiso social, en la que el agricultor espera recibir la mayor cantidad de beneficios de parte del Estado sin asumir el compromiso de emprender esfuerzos conjuntos que garanticen la obtención de objetivos propuestos, logrando estabilidad a los emprendimientos estatales. Los problemas mencionados anteriormente hacen que la agricultura se convierta en un sistema de trabajo con limitaciones que evitan su evolución.

Al iniciar el ciclo de siembra no cuenta con recursos financieros para adquirir paquetes tecnológicos de calidad, tampoco cuenta con adecuada mecanización de suelos ni sistemas de riego que aseguren un buen desarrollo agronómico del cultivo, lo que imposibilita una apta productividad.

Se agudiza el problema al no existir cadenas de comercialización que los beneficien, los grandes agricultores, aunque en minoría no son ajenos a estos problemas, que los empujan a vender parte de sus predios o maquinaria para poder captar liquidez cuando

ISSN: 1390-9320, Edición Especial, diciembre 2019 
ya se han cerrado las puertas en las entidades financieras. Como resultado, disminuye la fuerza laboral al disminuir su área de siembra y esto genera desempleo.

\section{REVISIÓN TEÓRICA}

El concepto de Seguridad Alimentaria surge en la década del 70 , basado en la producción y disponibilidad alimentaria a nivel global y nacional. En los años 80, se añadió la idea del acceso, tanto económico como físico. Y en la década del 90, se llegó al concepto actual que incorpora la inocuidad y las preferencias culturales, y se reafirma la Seguridad Alimentaria como un derecho humano (Honduras, 2013, p. 2).

Los conceptos de seguridad alimentaria que se referenciaron anteriormente han tenido una evolución en el tiempo, ya que, en los años ` 70 se hablaba de manejos de stocks y existencia de alimentos. En esos momentos, la FAO recomendaba que, para hacer frente a una emergencia, se debía tener 3 meses de consumo en existencias de los productos alimenticios básicos.

En los años '80, el concepto de Seguridad Alimentaria varía, enfocándose a la autosuficiencia, se pensaba que un país tenía seguridad alimentaria cuando podía producir todo lo que consumía, prácticamente era una visión autárquica. Si el país consumía todo lo que producía se protegía de la fluctuación de precios y la escasez.

En los años '90, después de la liberalización de los mercados, se asume que el problema no es solamente de disponibilidad de alimentos, es decir, puede haber abundancia de alimentos y, sin embargo, problemas de acceso a los mismos.

Así, en esta década se pone el énfasis en los problemas de acceso de los hogares porque, aunque un país tenga una alta producción su población puede padecer problemas de seguridad alimentaria y nutrición. De esta manera se ha llegado a un concepto amplio de seguridad alimentaria que tiene 4 pilares: la disponibilidad, la estabilidad, el acceso y la utilización.

Para el desarrollo de la investigación se emplearon diferentes métodos, tributando a una propuesta de política pública. Esta investigación va dirigida a los actores sociales que 
enmarcan el entorno específico de la provincia de Los Ríos, los cuales incluyen, tanto productores, dirigentes de gobiernos, como al propio ciudadano.

El período en el que se analiza será un análisis retrospectivo de 5 años desde el 2018 como año de cierre, para tener datos históricos que me permitan pronosticar las variables e indicadores que caractericen el objeto de estudio. Teniendo en cuenta las características socio-económicas del territorio objeto de estudio, el universo estará comprendido en el sector primario, específicamente en la actividad de Agricultura. Por su parte la muestra, atendiendo a las potencialidades endógenas, se enfocará en el análisis del sector bananero de la provincia Los Ríos, ya que, constituye uno de los principales rubros económicos dentro de la alimentación territorial y destinada a la exportación.

Las bases metodológicas para el diseño de la estrategia de seguridad alimentaria a nivel territorial, se desarrollaron en cuatro etapas, donde en la primera se define los objetivos que se persigue para poder diseñar la estrategia de seguridad alimentaria y la selección del grupo de trabajo, siguiendo con la etapa de diagnóstico estratégico de la seguridad alimentaria, identificando las dificultades principales asociadas a los componentes que fundamentan la seguridad alimentaria en un contexto local, siguiendo los siguientes pasos: Determinar las características fundamentales del sector objeto de estudio, Diseño muestral cuantitativo, Análisis de los cinco componentes que integran a la Seguridad Alimentaria, Identificación de los grupos en riesgo de inseguridad alimentaria, Identificación de las causas de los problemas alimentarios y nutricionales, Realización del análisis interno y externo. En la etapa tres se formuló las acciones estratégicas a partir del diagnóstico realizado del sector objeto de estudio y la población de la provincia Los Ríos, donde la principal herramienta de apoyo para la formulación es la caja de herramientas de Michel Godet (2000) y un modelo de un plan de acción, culminado con la etapa de evaluación y control, estableciendo un mecanismo de evaluación y control.

Se plasma resultados de los últimos cinco años entorno a la producción bananera, ya que como eje central de la investigación se utiliza el banano por ser la provincia de los Ríos una de las principales regiones productoras y exportadoras de este producto que constituye uno de los principales rubros de la economía nacional en el Ecuador. 
En la elaboración de dicha política entorno a la seguridad alimentaria se analizan los planes nacionales y locales de la economía en función de lograr el aseguramiento del acceso a los alimentos a todos los ciudadanos de la provincia Los Ríos. Proponiendo sobre la base de las características endógenas y los planes del buen vivir de la economía ecuatoriana, políticas a nivel de gobierno local o de territorio que permita el fortalecimiento de los cuatro pilares fundamentales que tiene la seguridad alimentaria: la disponibilidad, la estabilidad, el acceso y la utilización de los recursos alimenticios.

\section{ANÁLISIS DE LOS RESULTADOS}

En cuanto al destino de la producción agropecuaria, el 30\%, es para consumo externo y el $70 \%$ para consumo interno. Se abastece al mercado interprovincial. El arroz y la soya son materias primas para la elaboración de productos de consumo humano, el banano y cacao se destina para la exportación. Los productores se dedican mayoritariamente a la agricultura y solo aproximadamente un $10 \%$ combina sus actividades con la ganadería.

La pequeña agricultura campesina es importante porque permite la siembra de ciclo corto como las hortalizas, frutales, plantas medicinales y la producción pecuaria con la crianza de pollos y porcinos. Se estima que alcanza un 20,6\% predominando la agricultura de subsistencia que se observa en pequeños agricultores de cacao, arroz veranero, maíz y ganadería.

La pequeña agricultura campesina en esta provincia desarrolla principalmente cultivos de arroz, maíz, cacao y soya; en las organizaciones los socios tienen en promedio de tres a cinco hectáreas, pero se caracterizan por bajos niveles de producción y bajos ingresos. Dentro de los principales problemas que presentan aparece la falta de legalización de tenencia de tierra. La falta de tecnificación y maquinaria para los pequeños productores, así como también la implementación de más centros de acopio son los principales problemas estructurales que adolecen los agricultores. Sin dejar de mencionar una política de crédito que sea eficiente y oportuna.

Otro problema estructural es la escasa cultura de compromiso social, en la que el agricultor espera recibir la mayor cantidad de beneficios de parte del Estado sin asumir 
el compromiso de emprender esfuerzos conjuntos que garanticen la obtención de objetivos propuestos, logrando estabilidad a los emprendimientos estatales.

Los problemas mencionados anteriormente hacen que la agricultura se convierta en un sistema de pobreza del que el agricultor no termina de desligarse por completo. Al iniciar el ciclo de siembra no cuenta con recursos financieros para adquirir paquetes tecnológicos de calidad, tampoco cuenta con adecuada mecanización de suelos ni sistemas de riego que aseguren un buen desarrollo agronómico del cultivo, lo que imposibilita una apta productividad. Se agudiza el problema al no existir cadenas de comercialización que los beneficien.

El diagnóstico de la Seguridad Alimentaria y Nutricional. La seguridad alimentaria y nutricional es un factor definitivo en la calidad de vida de una población, pues está determinada por la disponibilidad, el consumo oportuno, la calidad y el aprovechamiento biológico de los alimentos. Este documento ofrece una herramienta metodológica con un enfoque integral que permite realizar el diagnóstico de la seguridad alimentaria y nutricional de una población.

Además, el documento brinda algunos elementos que se deben tener en cuenta a la hora de realizar dicho diagnóstico. Dentro del esquema, se proponen los siguientes procedimientos:

- Identificación y caracterización de los actores de acuerdo con los ejes temáticos de la seguridad alimentaria y nutricional;

- Revisión de documentación existente sobre los diferentes ejes;

- Diseño de los instrumentos para la recolección de información por medio de estudios descriptivos y métodos etnográficos;

- Sistematización de la información;

- Análisis de los hallazgos.

Identificación de los grupos en riesgo de inseguridad alimentaria 
El objetivo de este paso es identificar los grupos en riesgo de inseguridad alimentaria, o sea, los grupos de productores que, en forma permanente o temporal, tienen una producción insuficiente para cubrir las necesidades de la demanda territorial.

Sin embargo, es necesario precisar que existen otros grupos de riesgos que hacen alusión a la población y aquellas familias que tienen condiciones desfavorables, donde existen condiciones particulares que influyen en la intensidad del riesgo. La forma de distribución del ingreso, el nivel de ruralidad, el grado de marginalidad urbana y la existencia de grupos poblacionales marginados por razones étnicas o culturales, son elementos que determinan la mayor vulnerabilidad de ciertos grupos de esta índole que, que deben ser identificados a la hora de trazar estrategias.

En este sentido, es importante señalar que la identificación de los grupos en riesgo de inseguridad alimentaria, su caracterización económica, social y cultural permite la focalización de acciones en pro de la seguridad alimentaria en las personas que más lo necesitan, optimizando el uso de los recursos disponibles.

\section{Identificación de las causas de los problemas alimentarios y nutricionales}

El objetivo de este paso es la identificación de las causas de los problemas de inseguridad alimentaria, lo cual depende de la información disponible sobre los grupos en riesgo, del contacto que exista entre el municipio y la comunidad, de la relación del municipio con los servicios de la red social (municipalización o no de los servicios), de la capacidad técnica del municipio y de los recursos existentes.

Además, es importante señalar que los problemas relacionados con la inseguridad alimentaria, son el producto de la interacción de muchos elementos y consecuentemente, en su análisis debe incluirse un amplio espectro de factores culturales, sociales, político-administrativos, económicos y técnicos, aspecto fuertemente favorecido por el trabajo participativo.

\section{Realización del análisis interno}

El análisis interno tiene como objetivo identificar y evaluar las capacidades internas del territorio, es decir, las principales fortalezas (factores claves internos que favorecen el 
cumplimiento de la misión) y debilidades (factores internos claves que dificultan el cumplimiento de la misión) de la misma.

A partir de un estudio de la demanda de alimentos con 5 años como base de análisis y la producción del sector objeto de estudio, se establecerá, mediante un modelo de regresión, el comportamiento de las variables en post de proyectar los valores que sirvan de base en el desarrollo de una política de aseguramiento alimentario territorial.

Teniendo como primicia el hecho de que sabiendo lo que se demandará se podrá trabajar en el logro de la satisfacción de dicha demanda, apoyando el estado a la actividad productiva del sector.

\section{Realización del análisis externo}

El análisis externo tiene como objetivo identificar y evaluar las capacidades externas del macro entorno, es decir, las principales oportunidades y amenazas del entorno. En este sentido, es importante señalar que este análisis se hace tanto para el macro entorno actual como para el futuro. Además, para el análisis del macro entorno actual se valora lo que está sucediendo ahora y para el futuro.

\section{Formulación de las acciones estratégicas}

En el apartado, se formularán estrategias a partir del diagnóstico realizado del sector objeto de estudio y la población de la provincia Los Ríos, donde la principal herramienta de apoyo para la formulación es la caja de herramientas de Michel Godet (2000), donde se establecen los actores claves y variables claves para determinar a través de estrategias como deben influir unos con otros para lograr el objetivo fundamental que es el aseguramiento de la alimentación en la región de análisis.

\section{Análisis prospectivo de política agropecuaria}

Otra forma de enfrentar los problemas de escalas de producción es fomentar actividades económicas no agrícolas en los segmentos de agricultores más pequeños, que tienen dificultades estructurales para enfrentar los problemas de producción agrícola. Estas actividades normalmente son complementarias con las actividades agrícolas 
intraprediales, desde un punto de vista técnico y/o de uso de la fuerza de trabajo, lo que hace posible complementar los ingresos agrícolas que se generen en los predios, reforzando a veces la capacidad de inversión productiva dentro de ellos.

Esta línea de trabajo ha sido impulsada por el gobierno sectorial. El éxito alcanzado señala un camino a seguir. Sin embargo, debe tenerse presente que este tipo de emprendimientos requiere de asistencia técnica y de redes comerciales específicas para ser viables. Como se ha señalado, los factores de éxito de los emprendimientos asociativos son básicamente los mismos, ya sea que se trate de inversiones orientadas a resolver problemas de la producción primaria (por ejemplo, la administración de un tractor, de una obra común de riego o de otro tipo de infraestructura), ya sea que se trate de inversiones para lograr un escalamiento agroindustrial.

Aun así, en el documento se hace un análisis específico de este dilema en su dimensión de producción primaria, por cuanto la agricultura presenta una fuerte atomización de sus unidades productivas, lo cual obliga a buscar nuevos instrumentos para resolver los problemas de escala de producción.

\section{Resultado del objetivo de estudio}

La agricultura está actualmente siendo sometidas a una fuerte presión competitiva, que es consecuencia de la integración de la región a la economía nacional, en este contexto, aparece con fuerza la idea de que, para enfrentar esta presión en el caso de ciertos rubros y/o regiones productivas, es necesario un cambio tecnológico, concebido como un proceso de reconversión productiva. La reconversión productiva ha sido establecida como una de las cuatro líneas estratégicas de la política sectorial.

Sin embargo, las dificultades para obtener resultados hacen reflexionar acerca del alcance de esta línea de trabajo. La agricultura tiene regiones productivas de alto potencial productivo, cuyos productores pueden emigrar desde los actuales cultivos tradicionales hacia rubros más rentables y de mayor valor. El tema de la reconversión productiva adquiere connotaciones más complejas en áreas que tienen menos opciones productivas, ya sea porque presentan mayores limitaciones de suelo y clima o porque sencillamente no cuentan con rubros rentables hacia los cuales migrar.

ISSN: 1390-9320, Edición Especial, diciembre 2019 
En algunas regiones los programas de reconversión pueden tener impacto, mientras que en otras puede ser más conducente un enfoque distinto, que se denomina modernización productiva. A diferencia del primero, en que se trata de sustituir un rubro tradicional por otro nuevo, el enfoque de modernización apunta a valorizar y a potenciar el rubro tradicional, provocando cambios tecnológicos que lo hagan competitivo a nivel nacional e internacional. También pueden ser válidas las estrategias mixtas, que combinan en una misma región proyectos de reconversión productiva con proyectos de modernización.

Analizar el funcionamiento desde este punto de vista es clave para acceder a una mirada más agronómica acerca de sus posibilidades de impacto en las explotaciones de los agricultores.

- Riego: Los antecedentes disponibles señalan que en esta área se ha evolucionado en la línea correcta, pues es claro el alto impacto que tiene el riego en la productividad agrícola.

- Invernaderos: Ellos tienen un alto impacto en los ingresos brutos, lo que lo sitúa como el componente de inversión de mayor impacto dentro de la provincia. También es interesante el aporte de este componente en relación a los objetivos de reconversión productiva.

- Recuperación de Suelos: El gobierno sectorial apoya el mejoramiento de tierras de pastoreo, pero ello se hace principalmente a través de la introducción de infraestructura y equipos para el manejo de agostaderos, si consideramos además que el porcentaje restante de la inversión en tierras de pastoreo es básicamente entrega de semillas forrajeras, se concluye que existe un vacío pues no se está abordando los problemas de fertilidad de suelos. Este tema es estratégico, considerando el tamaño de los predios y la rotación intensiva de los suelos.

- Mejoramiento genético: Implementar esta línea de trabajo, en la medida en que interviene en la base genética del ganado, un área crítica que tiene un efecto sistémico sobre el manejo posterior del hato ganadero. Aún así, es necesario avanzar hacia una mejor focalización de estas inversiones en los segmentos menos avanzados, a fin de provocar cambios tecnológicos y no solo reponer semovientes de características similares a los preexistentes en las unidades de producción. 
- Infraestructura y mecanización: Al igual que respecto de otros tipos de maquinaria, cabe hacer una evaluación más fina sobre la real pertinencia de estas inversiones, pues normalmente ellas son de alto costo y tienen impactos aislados, que no alcanzan a generar un efecto sistémico en el sistema global de producción. Esta opinión se ve refrendada con los datos de la evaluación, en donde estos componentes son los que alcanzan un menor impacto sobre los ingresos brutos.

- Asistencia técnica y gestión: Esta área es clave para los agricultores y los resultados de las evaluaciones demuestran que cuando hay asistencia técnica de los procesos de inversión en general tienen mejores resultados. Sin embargo, se observa un avance discontinuo entre los diversos programas gubernamentales, razón por la cual se ha generado un vínculo entre los agricultores beneficiarios de programas con los servicios entregados por la empresa privada. Sin embargo, por diversas razones este esquema no ha entregado los resultados esperados.

\section{CONCLUSIONES}

Para contribuir al desarrollo económico de la Provincia de Los Ríos, se analizaron los aspectos teóricos relacionados con la seguridad alimentaria en el sector agrícola, se identificaron las políticas públicas agrícolas que tributan a la seguridad alimentaria en Ecuador, considerando los principales problemas que presentan como la falta de legalización de tenencia de tierra.

La falta de tecnificación y maquinaria para los pequeños productores, así como también la implementación de más centros de acopio son los principales problemas estructurales que adolecen los agricultores. Como resultado, disminuye la fuerza laboral al disminuir su área de siembra y esto genera desempleo.

Para esto se planteó una política de crédito que sea eficiente y oportuna. Creando un modelo de plan de acción y estrategias para la implementación de políticas públicas agrícolas encaminadas a garantizar la seguridad alimentaria de la provincia de Los Ríos; lo cual tiene suficiente pertinencia y conduce a mejorar la seguridad alimentaria, como objetivo estratégico de la administración pública en la provincia de Los Ríos.

ISSN: 1390-9320, Edición Especial, diciembre 2019 


\section{REFERENCIAS BIBLIOGRÁFICAS}

Banco Mundial. (2019). Pobreza y hambre: Problemas y opciones para la seguridad alimentaria en los países en desarrollo : La pobreza y el hambre: Temas y opciones sobre la seguridad alimentaria en los países en desarrollo. Recueprado de Documentos e informes del Banco Mundial: http://documentos.bancomundial.org/curated/es/604611468008104790/Laprobreza-y-el-hambre-temas-y-opciones-sobre-la-seguridad-alimentaria-en-lospaises-en-desarrollo

Bravo, D., Alvarado, R., \& Flores, B. (2015). Determinantes de la seguridad alimentaria en los hogares rurales del ecuador. Universidad De Las Américas, Facultad De Ciencias Económicas Y Administrativas, Quito.Recuperado De: Http://Dspace.Udla.Edu.Ec/Handle/33000/5379,

Calero, C., \& Molina, A. (2010). Mapa de la desnutrición crónica en el Ecuador. Quito: $M C D S / P M A$,

Chiriboga, M., \& Wallis, B. (2010). Diagnóstico de la pobreza rural en ecuador y respuestas de política pública. Grupo De Trabajo Sobre Pobreza Rural,

Domínguez-Serrano, M., \& El Rhomri, I. (2014). Límites del enfoque de la economía de los hogares: Una experiencia práctica de la ayuda alimentaria en el sahel. Paper presented at the Anales De Economía Aplicada 2014, 210-229.

Escribano, S. (2010). Situación y perspectivas de la soberanía alimentaria: Una revisión bibliográfica. Centro De Estudios Rurales Y De Agricultura Internacional,

Fao, F., Oms, P., \& Unicef. (2017). El estado de la seguridad alimentaria y la nutrición en el mundo 2017. Fomentando La Resiliencia En Aras De La Paz Y La Seguridad Alimentaria. Roma: FAO.

FAO. (mayo de 2019). Cumbre Mundial sobre la alimentación. Recuperado de http://www.fao.org/wfs/index_es.htm

FAO. (2019). Ecuador: Propuesta de estrategia nacional para mejorar la seguridad alimentaria. Recuperado de Políticas de seguridad alimentaria en los páises de 
la

Comunidad

Andina:

http://www.fao.org/tempref/GI/Reserved/FTP_FaoRlc/old/prior/segalim/pdf/mejo rar/cap5.pdf

García, C. (2012). Seguridad nutricional en crisis. Recuperado de http://digibuo.uniovi.es/dspace/bitstream/10651/4001/3/TFM_Garc\%C3\%ADaM az\%C3\%B3n.pdf

Jiménez Acosta, S. (2005). Seguridad alimentaria y nutricional: Una mirada global. Revista Cubana De Salud Pública, 31(3), 0-0.

Larrea, C., Torres, F. M., López, N. G., \& Rueda, M. B. C. (2007). Pueblos indígenas, desarrollo humano y discriminación en el Ecuador. Editorial Abya Yala.

LORSA. (2010). LEY ORGÁNICA DEL RÉGIMEN DE LA SOBERANÍA ALIMENTARIA. Recuperado de https://www.soberaniaalimentaria.gob.ec/pacha/wpcontent/uploads/2011/04/LORSA.pdf

Martinez, M. O. (13 de Septiembre de 2014). La seguridad alimentaria. Recuperado de http://derechoseguridadalimentaria.blogspot.com/

Ministerio de Agricultura y Ganadería. (2006). SEGUIMIENTO DE LA APLICACIÓN AL PLAN DE ACCION DE LA CUMBRE MUNDIAL SOBRE LA ALIMENTACION. INFORME NACIONAL. Ecuador. Recuperado el 2 de 2 de 2019, de www.fao.org/tempref/docrep/fao/Meeting/010/ag344s.pdfNutricional, S. A. (2011). Conceptos básicos. Programa Especial Para La Seguridad Alimentaria (PESA) En Centroamérica,

PESA. (2011). Seguridad alimentaria y nutricional: Conceptos básicos. pesa, centroamérica.(2011). Programa Especial Para La Seguridad Alimentaria (PESA) En Centroamérica, 19

Urdaneta, A. C. G., \& González, J. J. P. (2016). Marco conceptual de la medición de seguridad alimentaria (SA): Análisis comparativo y crítico de algunas métricas. Agroalimentaria, 22(43), 51-72. 
Villacís, B., \& Carrillo, D. (2011). Estadística demográfica en el ecuador: Diagnóstico y propuesta. Quito: Instituto Nacional De Estadística Y Censos, , 1-74.

WFP. Org. (2019). Programa mundial de alimentos. Retrieved from http://es.wfp.org/que-es-seguridad-alimentaria

Wong, S., \& Ludeña, C. (2006). Caracterización de la agricultura familiar en Ecuador. Superior ESPOL Ecuador. 